\title{
Prevention and management of tuberculosis in HIV positive patients living in countries with a low prevalence of Mycobacterium tuberculosis
}

\author{
M Gary Brook, Robert F Miller
}

We have re-examined the evidence on which current British Thoracic Society recommendations for primary and secondary prophylaxis and therapy of tuberculosis are based. We suggest that in a country such as the UK with a low prevalence of tuberculosis, primary prophylaxis should be offered primarily to tuberculin positive or anergic patients from high-incidence groups, including immigrants from high-prevalence countries, intravenous drug users and those with previous tuberculosis, that secondary prophylaxis be witheld from all but very high-risk patients and that four drug regimens which include ethambutol should be used for patients originating from, or who have lived in areas of the world with more than $2 \%$ primary isoniazid resistance.

(Genitourin Med 1996;72:89-92)

Keywords: Tuberculosis; HIV

\section{Introduction}

There is no doubt that tuberculosis (TB) is a major problem worldwide within the context of HIV but there are fewer data from countries with a low prevalence of tuberculosis, such as the UK, on which to base informed decisions. The British Thoracic Society has recently produced guidelines on the Control and prevention of tuberculosis in the United Kingdom ${ }^{1}$ which were published in the journal Thorax. In the previous month's issue there was a signed editorial from some of the members of the British Thoracic Society Tuberculosis sub-committee entitled Tuberculosis in the UK 1994; Current issues and future trends. ${ }^{2}$ Along with the 1992 British Thoracic Society's paper Guidelines on the management of tuberculosis and HIV infection in the United Kingdom ${ }^{3}$ these articles provide the British Thoracic Society's opinion on the management of tuberculosis in HIV positive patients in the UK, including case finding, contact tracing, chemoprophylaxis and use of BCG vaccination. They will also act as a source of information for physicans working outside the UK who may well be influenced by these recommendations. In this article we look critically at currently available information and offer an alternative interpretation of the data with particular reference to countries with a low prevalence of tuberculosis.

Camden \& Islington Community Health Services NHS Trust, London UK

Department of

Medicine

M G Brook

R F Miller

University College

London Medical School

R F Miller

Address correspondence to: Dr M G Brook, Departmen of Genitourinary Medicine, of Genitourinary Medicine

Mortimer Market Cen

Mortimer Market, Off

Wapper Street, Lon

Accepted for publication

19 September 1995
TB. However, Haiti is a country with a high prevalence of TB and a low rate of BCG vaccination in the adult population and taken together with the facts that a high proportion of the trial subjects were women $(77 \%)$ and follow-up of participants was variable (3-60 months), it makes these data difficult to interpret and apply to conditions where TB is less prevalent and where the majority of patients are men.

Primary isoniazid prophylaxis has also been successful in other countries with a high prevalence of TB including Spain, Italy and the US, although results have been less dramatic and the studies sometimes less well designed..$^{5-9}$ The crucial elements for successful primary prophylaxis include a population with a significant risk for TB (which in the case of the US includes intravenous drug users) and a means of identifying such patients. A positive tuberculin test is the commonest method used to identify the patients who would benefit from prophylaxis ${ }^{48}$ and most studies define a positive tuberculin reaction in HIV positive patients as $5 \mathrm{~mm}$ induration after 5 tuberculin units of PPD based on recommendations from the American Thoracic Society and Centers for Disease Control. ${ }^{10}$ However, it may be argued that given the propensity of HIV patients to become anergic (reduced evidence of cell-mediated immunity),,$^{7-9}$ any reaction to tuberculin might be regarded as positive. ${ }^{11}$ Tuberculin negative patients who are also anergic when tested with other recall antigens, such as Candida albicans and corynebacterium (for example the Merieux Multitest), in a high risk population and patients with a proven past history of clinical tuberculosis will also benefit from primary prophylaxis. ${ }^{7-9}$ What is not stated in most of these reports is the rate of drug side effects and ensuing withdrawal from the trials. There is considerable published information on the side effects of isoniazid in HIV-negative patients, where estimates of isoniazid hepatitis vary from 1 in 50 to 1 in 600 (depending on the definition), with reported mortalities of between 1 in 1000 and 1 in 4000 for all 
isoniazid users. ${ }^{12-15} \mathrm{~A}$ study of isoniazid prophylaxis in HIV positive patients in Italy reported that $35 \%$ of patients withdrew after less than one month for unspecified reasons and $39 \%$ of the rest were subsequently withdrawn because of side effects, atypical mycobacteriosis or death.$^{6}$ In two studies of tuberculosis therapy in HIV positive patients, $4-9 \%$ of treated patients had isoniazid-related hepatitis or rash severe enough to warrant discontinuation of the drug. ${ }^{16} 17$ There is no indication in these studies of patient characteristics that might identify those at particular risk of side effects but studies in HIV negative patients show that women (especially black women and those in the early post-partum period) and those patients aged $>35$ years or with high alcohol intake and preexisting liver disease have an increased rate of isoniazid hepatotoxicity. ${ }^{12} 1318$

Should primary isoniazid prophylaxis be offered to HIV-positive patients in the UK and other countries with a low prevalence of TB? The 1992 British Thoracic Society guidelines were in favour of primary prophylaxis and gave detailed instructions on how to identify suitable patients, ${ }^{3}$ although the advice in the 1994 paper has changed to that of the risks and benefits (of primary prophylaxis) remain to be determined. ${ }^{2}$ There are three main problems regarding the use of prophylaxis in countries with a low prevalence of TB. Firstly, where BCG immunisation has been used extensively, as in the UK, then tuberculin testing is far more difficult to interpret with regard to previous exposure to $\mathrm{TB}^{19}$ and there are no published data on the interpretation of tuberculin testing in BCG vaccinated HIV-positive patients. Identification of patients who might benefit from prophylaxis therefore becomes much more difficult. Secondly, there are few accurate data on the incidence of TB in the HIV positive population in the UK. One UK-based study suggested that $6 \%$ of HIV patients will develop TB but this estimate was based on a group of patients selected on the basis of either already having AIDS or who were in a cohort being studied for mycobacteria in stool. ${ }^{20} \mathrm{~A}$ report from the Communicable Diseases Surveilance Centre using data that are incomplete (because of the under-reporting of AIDS and the failure to report tuberculosis unless it was the first AIDSdefining condition), reported a $4.6 \%$ incidence tuberculosis in AIDS patients. ${ }^{19}$ There are no prospective data in the UK of HIV testing in TB patients although the national survey of 1993-4 may provide this information when published. Thirdly, the published studies of isoniazid prophylaxis were in populations with a prevalence of tuberculosis (evidence of previous exposure) of between $20 \%$ and $50 \%$. There are no such studies in populations with a lower background prevalence, such as the UK.

Given the low prevalence of TB, the uncertainties surrounding the interpretation of the tuberculin reaction and the rate of isoniazidrelated side effects, there appears to be little evidence to support a policy of routine screening and primary prophylaxis in HIV-positive patients born in countries with a low rate of TB, especially for patients who have had BCG vaccination. Patients who may benefit from a screening and prophylaxis programme, if the $4-8 \%$ risk of severe isoniazid toxicity is acceptable, would be immigrants from countries with a prevalence of tuberculosis, including people who have lived or worked there for prolonged periods ( $>6$ months), those with a past history of tuberculosis and injection drug users. ${ }^{519}$

\section{Diagnosis and Management of \\ Tuberculosis}

Much has been written about the spectrum of disease caused by $M$ Tuberculosis in HIV infected individuals. ${ }^{161721-24}$ In summary, the presentation of pulmonary tuberculosis that is typical of disease in non-HIV-infected patients with fever, cough and haemoptysis and apical/cavitatory disease on chest radiography is seen much less commonly in HIV-positive patients and is largely restricted to those with higher CD4+ lymphocyte counts. There is a greater incidence of extra-pulmonary disease in HIV-positive patients, occurring in approximately $60 \%$, a third of whom will also have pulmonary disease. ${ }^{1622}$ Pulmonary parenchymal disease is often widespread and interstitial. ${ }^{1721}$ The chest radiograph may be normal in between $5 \%$ and $20 \%$ of pulmonary disease. ${ }^{21}$ There is a much lower rate of sputum positivity for acid fast bacilli in pulmonary disease $(60 \%)$ and so the diagnosis is more frequently confirmed by bronchoscopy, culture and histology. ${ }^{16172122}$ There is a much higher mortality rate for HIV infected patients with TB as compared with HIV-negative patients. Small et al reported that $44 \%$ of 132 patients with HIV and TB died before their TB therapy was completed, but the excess deaths were usually related to other AIDS-related problems, with only $6.4 \%$ dying from TB. ${ }^{16}$

Response to standard anti-mycobacterial chemotherapy is very good with a rate of resolution of clinical features and loss of sputum positivity comparable with that seen in in HIV-negative patients ${ }^{161725}$ The rate of relapse after standard therapy is also very low and therefore there is no need to extend therapy beyond six months. In one study, relapses were only seen after inadequate therapy due to patient non-compliance and occured in 5\% of patients, all relapses being detected within 3 months of cessation of therapy. ${ }^{16}$

What is standard chemotherapy? The tuberculosis sub-committee of the British Thoracic Society suggest a triple regimen of rifampicin, isoniazid and pyrazinamide given for two months, followed by rifampicin and isoniazid for a further four months, with the addition of ethambutol in the first two months only if there is a significant rate of drug resistance locally or the patient has been previously treated abroad. ${ }^{23}$ However, there are no studies comparing quadruple with triple drug therapy in HIV infected individuals with tuberculosis and there is ample evidence of the propensity of $M$ tuberculosis to develop drug resistance or to be drug resistant within the context of HIV infection, as seen in New York and Florida. ${ }^{26}{ }^{27} \mathrm{We}$ therefore agree that triple therapy should be standard for HIV positive patients with TB with the additional proviso to the Brirish Thoracic 
Society guidelines that quadruple therapy should be instituted in those patients who come from countries where the prevalence of primary isoniazid resistance is significant (that is, $>2 \%$ ): in effect this means patients from Southern Europe, Africa, SE Asia, the Indian sub-continent, South America and parts of the United States (for example New York).

\section{Secondary Chemo-prophylaxis for TB}

Both the American and British Thoracic Societies recommend secondary isoniazid prophylaxis for life following the successful treatment of tuberculosis. ${ }^{329}$ However, these recommendations are not supported by reference to published research in this area. Indeed, we are unable to identify any work that supports this advice. There are, in fact, very good arguments against this proposal. Firstly, the reported rate of relapse after optimal therapy is very low and the quoted overall relapse rate of $5 \%$ includes patients with sub-optimal treatment. ${ }^{16}$ Secondly, the median survival of HIVpositive patients with tuberculosis is between 7 and 16 months, suggesting that most patients die of other causes before there is time for any potential late relapse. ${ }^{1617} \mathrm{We}$ suggest that secondary prophylaxis should only be offered if there are compelling reasons, for instance if relapse of a patients tuberculosis would pose a serious threat to other immunocompromised people within the same household. However, all patients should be closely followed by clinical monitoring and chest radiography after treatment of their tuberculosis to detect relapse, especially if there are doubts about the completeness of the patient's drug compliance.

\section{Which Patients Should Be Isolated?}

The British Thoracic Society guidelines suggest that if an HIV-positive patient is hospitalised with known or suspected TB they should be isolated at least until the sputum smear microscopy for acid fast bacilli is known. Patients should continue in isolation if the sputum smear is positive until they have received two weeks anti-tuberculous therapy. The guidelines also suggest that patients with pulmonary TB diagnosed by bronchoscopy and broncho-alveolar lavage (BAL) but who are sputum smear negative and patients with isolated extra-pulmonary TB need not be isolated ${ }^{1}$ Whilst this advice is perfectly sound for immunocompetent patients, there are several pieces of evidence suggesting that this may not be true for specialist in-patient units treating HIV-positive patients. HIV-infected-patients seem to be more susceptible to developing clinical infection after apparent minimal exposure to $M$ tuberculosis. For instance, one study describes an HIV-positive man who had a normal chest radiograph and negative microscopy of bronchoscopic lung biopsy specimens who was subsequently diagnosed as having pulmonary tuberculosis by culture of lung biopsy specimens after spending a month on an HIV ward. Of eighteen other HIV-positive patients who came into close contact with this patient, eight subsequently developed TB; seven of these within two months. ${ }^{30}$ Another report shows that exposure to patients with smear-positive tuberculosis need only be brief for HIV positive-patients to become infected. In that study a case cluster of tuberculosis was caused by a single strain of $M$ tuberculosis, where the index case was only briefly in contact on the same ward with the two patients who were subsequently infected. ${ }^{31}$ Recent studies also reveal that approximately $60 \%$ of tuberculosis in HIV-positive patients in the US is due to secondary infection rather than reactivation whereas only 10 to $20 \%$ of tuberculosis in nonHIV-infected adults in developed countries is due to secondary infection. ${ }^{32}{ }^{33}$ In the light of this evidence of increased susceptibility of HIVpositive patients to developing active tuberculosis after minimal exposure to $M$ Tuberculosis, we suggest that as well as isolating all patients with pulmonary tuberculosis diagnosed by sputum microscopy, those diagnosed by microscopy of BAL fluid should also be isolated in a sideroom if there are other HIV-positive patients on the ward, until they have received at least two weeks anti-tuberculosis therapy.

We also have a duty of responsibility to HIVinfected health care workers who are also at significant risk of acquiring tuberculosis when caring for infected patients. National guidelines for HIV-infected health care workers advise that they be given duties that would not put them in a position where they pose an infectious risk to their patients or where the patient may transmit infection to the member of staff. However, it is likely that there are HIV-infected health care workers who either do not know they are infected or who choose to withhold this information. Staff education about the risks of acquisition of tuberculosis is therefore very important, as is the provision of occupational health advice given on the basis of high regard for the needs of the staff member.

The threat of cross infection is not confined to the ward. Vigilence towards identifying and isolating infectious and potentially infectious patients should be practised in the out-patients area as was demonstrated by an outbreak in a Miami hospital where twenty two HIV-positive patients were found to have acquired multiresistant TB whilst attending the same outpatient clinic as an HIV-infected individual with tuberculosis. ${ }^{34}$

\section{Notification of Tuberculosis}

Tuberculosis is a notifiable disease and in most developed countries doctors have a statutory obligation to report all cases to the proper authorities (the local Consultant in Communicable Disease Control in England and Wales and the Chief Administrative Medical Officer in Scotland). However, on the basis of "off the record" information gained from colleagues, we believe that many cases in HIV-positive patients are not reported. Part of the reason for under-reporting of tuberculosis in HIV-positive patients is the perception by some physicians that it would be a breach of confidentiality. Only by thorough reporting can it be ensured that appropriate contact tracing is performed and it is essential that there are ade- 
quate statistics for epidemiological planning on a national level. ${ }^{19} 28$ Public health aspects of notification therefore outweigh any perceived threat to individual patient confidentiality. The patient's right to privacy will not be affected as public health doctors have a duty of confidentiality with regard to the patient's HIV status. Additionally, it is especially important that HIV-positive patients should be dealt with sensitively during the process of contact tracing.

\section{Conclusion}

The extent of the problem of tuberculosis in HIV-infected patients in the UK is not known and much of the current management guidelines are based on extrapolations of data from other countries. Careful review suggests that there are few published data to support many of the recommendations made by the British Thoracic Society for the management of HIVinfected individuals in the UK who have $M$ tuberculosis infection. Based on current knowlege we suggest the following strategy may be appropriate: 1) Primary chemoprophylaxis should be offered only to patients who are tuberculin positive or anergic (see definition above) and who are also either immigrants from countries with a high prevalence of tuberculosis or who have spent at least six months in such countries or who have a past history of tuberculosis or are intravenous drug users. 2) All sputum smear-positive patients should be isolated as should all BAL fluid microscopy positive patients if there are other HIV-positive patients on the ward. 3) Active infection should be treated with three drugs for two months (usually rifampicin, isoniazid, pyrazinamide) with the addition of ethambutol for patients at risk of having an isoniazid-resistant organism followed by rifampicin and isoniazid for four more months providing the organism is not resistant to any of these antibiotics. 4) Following successful treatment of active disease secondary prophylaxis is not recommended except in exceptional circumstances. However, all patients should be closely monitored both clinically and radiographically for possible subsequent relapse. 5) All HIV-positive patients with tuberculosis must be notified to the public health authorities to enable contact tracing to take place and to provide epidemiological data.

It is important to remember that tuberculosis in HIV positive patients may be difficult to diagnose because of a higher rate of sputumnegative cases and because of clinical and radiographic atypical presentations.

We are grateful to Drs Anton Pozniak, Kevin De Cock, Geoff Scott and Janet Darbyshire for their critical review of the manuscript.

1 Joint Tuberculosis Committee of the British Thoracic Society. Control and prevention of tuberculosis in the United Kingdom: code of practice 1994. Thorax 1994 49:1193-200.

2 Ormerod LP, Shaw RJ, Mitchell DM. Tuberculosis in the UK, 1994: current issues and future trends. Thorax 1994; 49:1085-9.
3 Subcommittee of the Joint Tuberculosis Committee of the British Thoracic Society. Guidelines on the management of tuberculosis and HIV infection in the United Kingdom. BMF 1992;304:1231-3.

4 Pape JW, Jean SS, Ho JL, Hafner A, Johnson WD. Effect of isoniazid prophylaxis on incidence of active tuberculosis and progression of HIV infection. Lancet 1993;342: 268-72.

5 Selwyn PA, Hartel D, Lewis VA. Prospective study of the risk of tuberculosis among intravenous drug users with human immunodeficiency virus infection. $N$ Engl Med $f$ 1989;320:545-50.

6 Bevilacqua N, Marasca G, Moscati A, Fantoni M, Ricci F, Ortona L. Tuberculosis and HIV infection. Lancet 1993;342:677.

7 Guelar A, Gatell JM, Verdejo J, et al. A prospective study of the risk of tuberculosis among HIV-infected parients. AIDS 1993;7:1345-9.

8 Moreno S, Baraia-Etxaburu J, Bouza E, et al. Risk for developing tuberculosis among anergic patients infected with HIV. Ann Intern Med 1993;119:194-8.

9 Barrio E, Carballo E, Cabarcos A. Prevalence of tuberculosis and the use of isoniazid prophylaxis in anergic HIVinfected individuals. AIDS 1994;8:857-8.

10 American Thoracic Society and Centers for Disease Control. Diagnostic standards and classification of tuberculosis. $A m$ Rev Resp Dis 1990;142:725-35.

11 Graham NH, Nelson KE, Solomon $\mathrm{L}$, et al. Prevalence of tuberculin positivity and skin test anergy in HIV-1seropositive and -seronegative intravenous drug users. seropositive and -serone

12 Snider DE, Caras GJ. Isoniazid-associated hepatitis deaths: a review of available information. Am Rev Respir Dis 1992 145:494-7.

13 Anon. Severe isoniazid-associated hepatitis-New York, 1991-3. MMWR 1993;42:545-7.

14 Moulding T. Isoniazid associated hepatitis deaths: a review of available information. Am Rev Respir Dis 1992;145: 1643-4.

15 Derby LE, Gutthan SP, Jick H, Dean AD. Liver disorders in patients receiving chlorpromazine or isoniazid. Pharmacotherapy 1993;13:353-8.

16 Small PM, Schecter GF, Goodman PC, Cande MA Chaisson RE, Hopewell PC. Treatment of tuberculosis in patients with advanced human immunodeficiency virus patients with advanced human immuno

17 Chaisson RE Schecter GF, Theuer CP, Rutherford GW, Echenberg DF, Hopewell PC. Tuberculosis in patients with the acquired immunodeficiendy syndrome. Am Rev Respir Dis 1987;136:570-4.

18 Wu JC, Lee SD, Yeh PF, et al. Isoniazid-rifampin-associated hepatitis in hepatitis B carriers. Gastroenterology. 1990 98:502-4.

19 Watson JM, Meredith SK, Whitmore-Overton E, Bannister B, Darbyshire JH. Tuberculosis and HIV: estimates of the overlap in England and Wales. Thorax 1993;48: 199-203.

20 Helbert M, Robinson D, Buchanan D et al. Mycobacteria infection in patients infected with the human immunodefiinfection in patients infected with the
ciency virus. Thorax 1990;45:45-8.

21 Laguna F, Adrados M, Diaz F, et al. AIDS and tuberculosis in Spain. A report of 140 cases. J Infect 1991;23:139-44.

22 Foley NM, Miller R. Tuberculosis and AIDS: is the white plague up and coming? F Infect 1993;26:39-43.

23 Barnes PF, Bloch AB, Davidson PT, Snider DE. Tuberculosis in patients with human immunodeficiency virus. N Engl f Med 1991;324:1644-50.

24 Mitchell DM, Miller RF. Recent developments in the management of the pulmonary complications of HIV disease. Thorax 1992;47:381-90.

25 Brindle RJ, Nunn PP, Githui W, Allen BW, Gathua S, Waiyaki P. Quantitative bacillary response to treatment in HIV-associated pulmonary tuberculosis. Am Rev Respir Dis 1993;147:958-61.

26 Frieden TR Sterling T, Pablos-Mendez A, Kilburn JO, Cauthen GM, Dooley SW. The emergence of drug resistant tuberculosis in New York City. $N$ Engl f Med 1993;328:521-6.

27 Fischl MA, Daikos GL, Uttamchandani RB, et al. Clinica presentation and outcome of patients with HIV infection and tuberculosis caused by multi-drug-resistant bacilli. Ann Int Med 1992;117:184-90.

28 Davies PDO. Tuberculosis and HIV: blind man's buff. Thorax 1993;48:193-4.

29 American Thoracic Society. Mycobacterioses and the acquired immunodeficiency syndrome. Am Rev Respir Dis 1987;141:492-5

30 Di Perri G, Danzi MC, De Checchi G, et al. Nosocomial epidemic of active tuberculosis among HIV-infected patients. Lancet 1989;ii:1502-4.

31 Kent RJ, Uttley AHC, Stoker NG, Miller R, Pozniak AL Transmission of tuberculosis in a British centre for Transmission of tuberculosis in a British centre

32 Small PM, Hopewell PC, Singh SP, et al. The epidemiolog of tuberculosis in San Francisco. N Engl $\mathfrak{f}$ Med 1994;330: 1703-9.

33 Alland D, Kalkut GE, Moss AR, et al. Transmission of tuberculosis in New York. $N$ Engl $; f$ Med 1994;330 1710-16.

34 Fischl MA, Uttamchandani RB, Daikos GL et al. An outbreak of tuberculosis caused by multiple-drug-resistant tubercle bacilli among patients with HIV infection. Ann Int Med 1992;117:177-83. 